\title{
Interval to Biochemical Failure as a Biomarker for Cause-Specific and Overall Survival After Dose-Escalated External Beam Radiation Therapy for Prostate Cancer
}

\author{
Nirav S. Kapadia, MD; Karin Olson, PhD'; Howard M. Sandler, MD²; Felix Y. Feng, MD³; and Daniel A. Hamstra, MD, PhD
}

BACKGROUND: After external beam radiation therapy (EBRT) for prostate cancer, a short interval to biochemical failure of $<18$ months has been proposed as a surrogate for cause-specific survival. Because EBRT dose influences biochemical failure, the authors investigated the interval to biochemical failure in a cohort of patients treated with dose-escalated EBRT. METHODS: From 1998 to 2008, 710 patients were treated with EBRT ( $\geq 75$ grays) \pm androgen deprivation therapy (ADT) at the University of Michigan. Biochemical failure was defined using the Phoenix consensus definition (nadir $+2 \mathrm{ng} / \mathrm{mL}$ ). A short interval to biochemical failure was defined as $<18$ months after completing radiotherapy and/or ADT. The associations between biochemical failure, the interval to biochemical failure, and clinical factors with cause-specific survival (CSS) and overall survival (OS) were evaluated. RESULTS: There were 149 biochemical failures (21\%), and short interval to biochemical failure accounted for $14 \%$ and $40 \%$ of biochemical failures in those with intermediate-risk or high-risk disease, respectively. Biochemical failure impacted CSS $(P<.0001)$ but not OS $(P=.36)$. However, a short interval to biochemical failure predicted decreased CSS $(P<.0001$; hazard ratio [HR], 5.6; 95\% confidence interval [Cl], 2.4-13.0) and OS ( $P<.0001 ; \mathrm{HR}, 4.8 ; 95 \% \mathrm{Cl}, 2.3-10.3)$ when compared with a long interval to biochemical failure. The 8 -year OS was $78 \%$ without biochemical failure, compared with $87 \%$ with a long interval to biochemical failure $(P=.1 ; \mathrm{HR}, 0.7 ; 95 \% \mathrm{Cl}, 0.4-1.1)$ and $38 \%$ with a short interval to biochemical failure $(P<$ .0001; HR, 3.7; $95 \% \mathrm{Cl}, 2.3-5.9)$. On multivariate analysis, a short interval to biochemical failure increased the risk of prostate cancer death $(P<.0001 ; \mathrm{HR}, 18.1 ; 95 \% \mathrm{Cl}, 8.4-39)$ and all cause mortality $(P=.0027 ; \mathrm{HR}, 1.5 ; 95 \% \mathrm{Cl}, 1.2-2.1)$, whereas a long interval to biochemical failure did not. CONCLUSIONS: The relation between the interval to biochemical failure, CSS, and OS was independently validated in patients treated with dose-escalated EBRT. Further evaluation of the interval to biochemical failure as a surrogate endpoint is warranted. Cancer 2012;118:2059-68. (C) 2011 American Cancer Society.

KEYWORDS: risk factors, surrogate, prostate-specific antigen, cause-specific survival, biomarker.

\section{INTRODUCTION}

The long natural history of prostate cancer (PCa) has led to the search for biomarkers to be used as surrogates for either cause-specific survival (CSS) or overall survival (OS). After prostatectomy, the presence of detectable prostate-specific antigen (PSA) is defined as biochemical failure, whereas classifying biochemical failure after external beam radiation therapy (EBRT) is more complex, because residual normal prostatic tissue remains, and the PSA remains detectable. The current consensus (Phoenix) definition of biochemical failure after EBRT or brachytherapy with or without androgen deprivation therapy (ADT) is when post-treatment PSA reaches at least $2 \mathrm{ng} / \mathrm{mL}$ greater than its lowest post-treatment (or nadir) value. ${ }^{1}$ However, biochemical failure by itself does not necessarily portend a grave prognosis, a fact recognized both by an American Society of Therapeutic Radiation Oncology consensus panel and the National Comprehensive Cancer

Corresponding author: Daniel A. Hamstra, MD, PhD and Felix Y. Feng, MD, University of Michigan Health System, 1500 East Medical Center Drive, Floor B2, Room C490, Ann Arbor, Ml 48109-5010; Fax: (734) 763-7370; dhamm@med.umich.edu

${ }^{1}$ University of Michigan Medical Center, Ann Arbor, Michigan; ${ }^{2}$ Cedars Sinai Medical System, Los Angeles, California; ${ }^{3}$ Ann Arbor Veteran Affairs Medical System, Ann Arbor, Michigan

F.Y.F. and D.A.H. contributed equally to this article.

DOI: 10.1002/cncr.26498, Received: May 11, 2011; Revised: June 29, 2011; Accepted: July 18, 2011, Published online August 26, 2011 in Wiley Online Library (wileyonlinelibrary.com) 
Network (NCCN) guidelines. ${ }^{2,3}$ In fact, given the lack of clearly proven benefit of early salvage ADT, these bodies recommend that clinical failure-not biochemical failure-indicates the need for salvage ADT.

Because of the poor specificity of biochemical failure for later clinical failure, several other metrics have been evaluated as potential surrogates for clinical trial design or by which to plan the use of salvage therapy. The level and timing of PSA nadir have both been correlated with biochemical failure, distant metastasis (DM), and CSS in radiotherapy (RT) series ${ }^{4-9}$; both PSA velocity and/or doubling time $\mathrm{e}^{10-12}$ have also been evaluated as potential surrogates. However, each of these has been demonstrated as an incomplete surrogate for CSS, with even worse correlation with OS. The interval to biochemical failure has also been evaluated as a surrogate for DM and CSS in both surgical and RT series. ${ }^{13,14}$ Investigators at Fox Chase Cancer Center evaluated the time from the end of all therapy (EBRT and ADT) to biochemical failure, and identified 18 months as a cutpoint that was optimal as a potential surrogate for CSS. ${ }^{14}$ This cutpoint was subsequently evaluated in a multi-institutional series and found to be superior to both PSA nadir and PSA doubling time as a surrogate for CSS. ${ }^{15}$ Analysis of the TROG 96.01 randomized trial also established an interval to biochemical failure of $<24$ months as a potential surrogate for CSS among patients randomized to low-dose (66 grays [Gy]) RT alone or with either 3 or 6 months of ADT. ${ }^{16}$ These studies all used either uniform low-dose $\mathrm{RT}^{16}$ or a broad range of RT doses, ${ }^{14,15}$ but because the level and timing of the PSA nadir as well as biochemical failure are functions of RT dose, this might influence the prognostic significance of the interval to biochemical failure. Therefore, we sought to validate the interval to biochemical failure in a cohort of patients uniformly treated with dose-escalated RT.

\section{MATERIALS AND METHODS}

\section{Patient Selection}

Between 1998 and 2008, 718 PCa patients were treated with dose-escalated EBRT \pm ADT at the University of Michigan Medical Center (Ann Arbor, Mich). Information about the interval to biochemical failure was available for $99 \%$ (710 of 718 ) of patients, who form the cohort for this analysis.

\section{Informed Consent}

This was a retrospective review that was institutional review board-approved and not deemed to require informed consent. However, from 2002 to the present, at the time of radiation treatment patients have signed informed consent to have their clinical information included in the prostate database at the University of Michigan Medical Center.

\section{Staging}

All patients were clinically staged per the American Joint Committee on Cancer sixth edition staging criteria into low-risk, intermediate-risk, and high-risk groups. Patients with high-risk disease routinely underwent staging with computed tomography (CT) and bone scan to rule out metastatic disease. Per NCCN guidelines, staging was not part of standard practice for those with low-risk or intermediate-risk disease. In addition, neither endorectal coil or pelvic magnetic resonance imaging were routinely used. The Charlson Comorbidity Index was used to evaluate comorbid illness and was evaluable in 97\% (691 of 710) of patients. ${ }^{17}$

\section{Treatment}

Patients were treated based on CT planning with either 3dimensional conformal EBRT or intensity-modulated RT prescribed such that the $95 \%$ isodose surface encompassed the planning target volume (PTV). Low-risk patients were treated at the prostate alone, and intermediate-risk patients were routinely treated at the prostate and seminal vesicles. High-risk patients routinely (87\%) received treatment to the pelvic lymph nodes followed by a boost to the prostate and seminal vesicles. ADT was used in 39\% of all patients: $11 \%$ (median, 4 months), 27\% (median, 6 months), and 79\% (median, 21 months) for low-risk, intermediate-risk, and high-risk patients, respectively. For purposes of analysis, short-term ADT was defined as $<12$ months, whereas long-term ADT was defined as $\geq 12$ months.

\section{Follow-up and Endpoints}

Follow-up and PSA were routinely obtained at 3- to 4month intervals for the first 2 years, every 6 months for 5 years, and every 6 to 12 months thereafter. The Phoenix definition (nadir $+2 \mathrm{ng} / \mathrm{mL}$ ) was used to define biochemical failure ${ }^{1}$; DM was defined as any clinical, radiographic, or pathologic evidence of DM. OS was defined as death from any cause, whereas CSS was defined as 1) death attributed to $\mathrm{PCa}$ or death in any patient with either 2) castration-resistant $\mathrm{PCa}$ or 3) documented evidence of metastatic disease before death. The time of follow-up was based upon the last day of RT; however, the interval to biochemical failure was defined based upon the time 
Table 1. Clinical and Treatment-Related Factors

\begin{tabular}{|c|c|c|c|c|}
\hline \multirow[t]{2}{*}{ Variable } & \multirow[t]{2}{*}{ No Biochemical Failure } & \multicolumn{2}{|c|}{ Biochemical Failure } & \multirow[t]{2}{*}{$\boldsymbol{P}$} \\
\hline & & Short IBF & Long IBF & \\
\hline Number of patients & 563 & 41 & 106 & \\
\hline Age, median y (IQR) & $69.5(63-74)$ & $66.4(59-75)$ & $69.8(64-73)$ & $.5^{\mathrm{a}}$ \\
\hline CMI, mean & 0.80 & 0.74 & 0.61 & $.066^{\mathrm{a}}$ \\
\hline None & $55 \%$ & $64 \%$ & $47 \%$ & $.03^{\mathrm{b}}$ \\
\hline 1 & $24 \%$ & $18 \%$ & $37 \%$ & \\
\hline$\geq 2$ & $21 \%$ & $18 \%$ & $16 \%$ & \\
\hline PSA (IQR) & $7.8(5.3-12.1)$ & $26.1(12.4-48)$ & $11.3(6.0-24)$ & $<.001^{\mathrm{a}}$ \\
\hline \multicolumn{5}{|l|}{ Gleason score } \\
\hline $2-6$ & $39 \%$ & $2 \%$ & $25 \%$ & $<.0001^{\mathrm{b}}$ \\
\hline 7 & $45 \%$ & $37 \%$ & $51 \%$ & \\
\hline 8 & $9 \%$ & $22 \%$ & $18 \%$ & \\
\hline $9-10$ & $7 \%$ & $39 \%$ & $6 \%$ & \\
\hline Clinical TNM classification & & & & $<.001^{\mathrm{a}}$ \\
\hline T1-T2a & $77 \%$ & $31 \%$ & $57 \%$ & \\
\hline $\mathrm{T} 2 \mathrm{~b}-\mathrm{T} 2 \mathrm{c}$ & $17 \%$ & $20 \%$ & $20 \%$ & \\
\hline T3-T4 & $6 \%$ & $49 \%$ & $23 \%$ & \\
\hline \multicolumn{5}{|l|}{ Radiation therapy } \\
\hline Dose, median Gy (IQR) & $78(76-78)$ & $77(77-78)$ & 77 (76-78) & $.7^{\mathrm{a}}$ \\
\hline Pelvic EBRT & $23 \%$ & $56 \%$ & $39 \%$ & $<.0001^{\mathrm{b}}$ \\
\hline ADT & & & & $.004^{\mathrm{b}}$ \\
\hline No & $63 \%$ & $39 \%$ & $53 \%$ & \\
\hline Yes & $37 \%$ & $61 \%$ & $47 \%$ & \\
\hline Duration, mo & $6.8(6.0-24.3)$ & $24.9(6.2-31)$ & $6.4(6.0-20.4)$ & $<.001^{\mathrm{a}}$ \\
\hline NCCN risk group & & & & $<.0001^{\mathrm{b}}$ \\
\hline Low & $26 \%$ & $0 \%$ & $12 \%$ & \\
\hline Intermediate & $48 \%$ & $17 \%$ & $40 \%$ & \\
\hline High & $26 \%$ & $83 \%$ & $48 \%$ & \\
\hline
\end{tabular}

Abbreviations: ADT, androgen deprivation therapy; CMI, Charlson Comorbidity Index; EBRT, external beam radiation therapy; Gy, grays; IBF, interval to biochemical failure; IQR, interquartile range; NCCN, National Comprehensive Cancer Network; PSA, prostate-specific antigen.

${ }^{a}$ Analysis of variance.

${ }^{\mathrm{b}}$ Chi-square.

from the end of all therapy (RT or ADT) to biochemical failure. Those who progressed while still on ADT were scored as having an interval to biochemical failure of zero.

\section{Statistical Analysis}

Comparison between continuous variables was performed with 1-way analysis of variance, whereas comparisons between categorical variables used the chi-square test. The log-rank test and Kaplan-Meier methods compared the influence of single variables on survival endpoints, whereas multivariate analyses were conducted using stepwise Cox proportional hazard models.

\section{RESULTS}

\section{Patient Characteristics and Follow-up}

Median age at the time of treatment was 69 years (interquartile range [IQR], 63-74), with no difference in age by biochemical failure status (none, a short interval to biochemical failure $[<18$ months from the end of all therapy], or long interval to biochemical failure, Table 1). Median follow-up was 64 months (IQR, 36-89): 57 months (IQR, 34-82) in those without biochemical failure, 69 months (IQR, 35-91) in those with a short interval to biochemical failure, and 98 months (IQR, 71-131) in those with long interval to biochemical failure. Not surprisingly, patients with a short interval to biochemical failure were more likely to have higher-risk disease as measured by PSA, TNM classification, Gleason score, and risk group in comparison to those without biochemical failure or with a long interval to biochemical failure (Table 1).

\section{Biochemical Failure and Clinical Outcome}

Biochemical failure was observed in 147 patients $(21 \%$ of all) at a median of 42 months (IQR, 22-67) from the end 

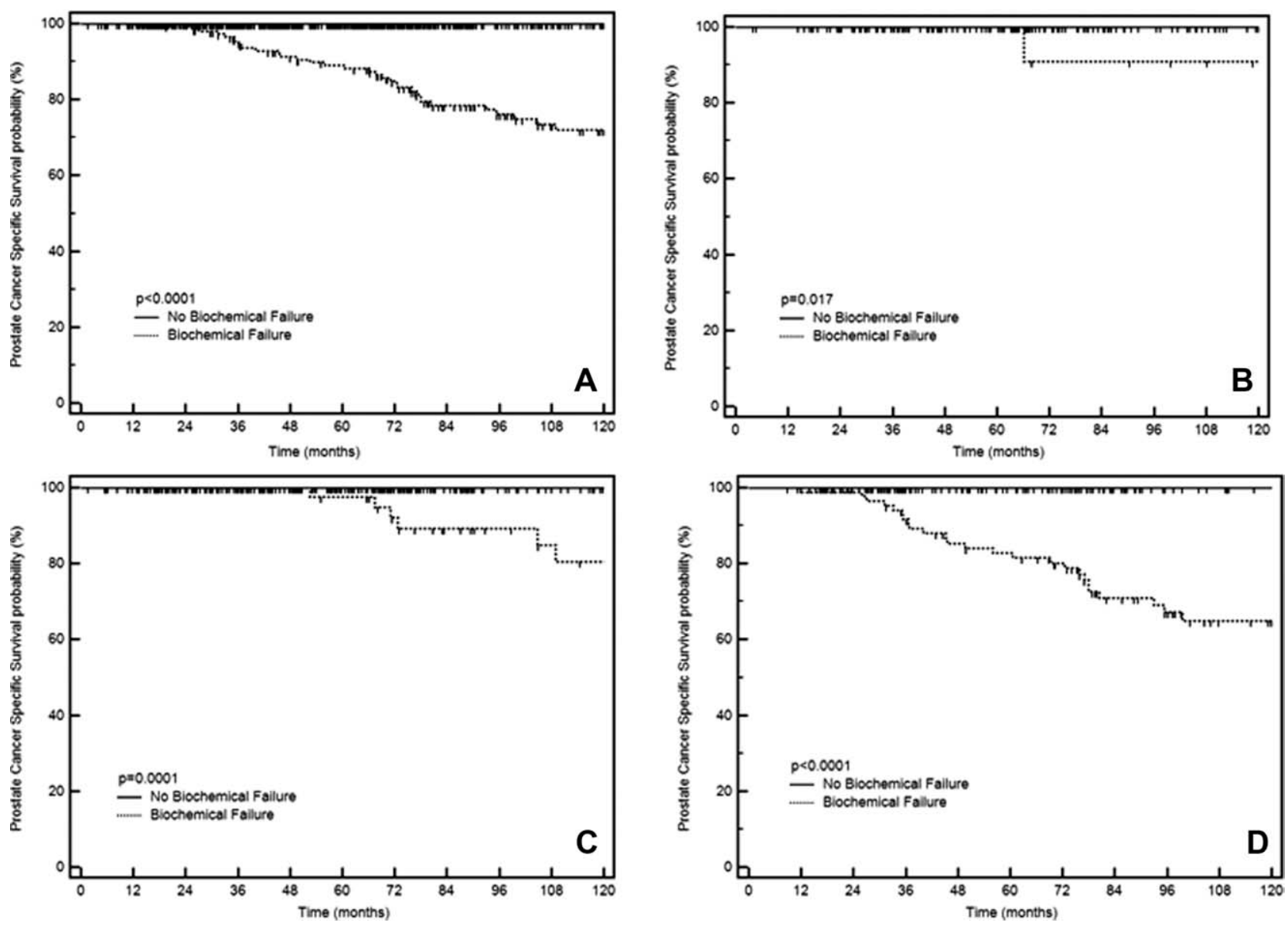

Figure 1. Biochemical failure predicts for worse cause-specific survival among all patients (A), and as stratified by National Comprehensive Cancer Network risk group (low risk, B; intermediate risk, C; high risk, D).

of EBRT. A short interval to biochemical failure was seen in 41 patients, occurring a median 13 months (IQR, 822) from the end of RT, whereas for those with a long interval to biochemical failure this occurred a median 56 months (IQR, 40-80) after completing RT. Among the 160 low-risk patients, there were 13 biochemical failures $(8 \%)$ and no patients with a short interval to biochemical failure. Among 319 patients with intermediate-risk disease, there were 49 biochemical failures (15\%), and 7 of these were with a short interval to biochemical failure (2\% of all intermediate-risk patients and $15 \%$ of all biochemical failures in this group). Among the 231 high-risk patients, there were 85 biochemical failures (36\%), and 34 of these had a short interval to biochemical failure (15\% of all high-risk patients and $40 \%$ of biochemical failures in this group). The rates of PCa death ( \pm standard error of the mean) were greater with increasing risk: $1 \%$ $( \pm 1 \%), 3 \%( \pm 1 \%)$, and $15 \%( \pm 5 \%)$ at 8 years for lowrisk, intermediate-risk, and high-risk patients, respectively
$(P<.0001)$. No patient died of PCa without first experiencing biochemical failure. As a result, cause-specific survival was worse in those with biochemical failure for the whole population $(P<.0001)$ and when broken down by NCCN risk groups (Fig. 1). However, the positive predictive value of biochemical failure was low, as to date only $24 \%$ (36 of 147) of patients with biochemical failure have died of PCa. In addition, there was no correlation between biochemical failure and OS $(P=.38)$ even for patients with high-risk disease $(P=.8$, Fig. 2$)$.

\section{Interval to Biochemical Failure and Clinical Outcome}

Because biochemical failure was poorly correlated with CSS and OS, the impact of a short interval to biochemical failure for subsequent clinical events was evaluated. At 8 years, patients with a long interval to biochemical failure were less likely to experience metastasis, as compared with those with a short interval to biochemical failure 

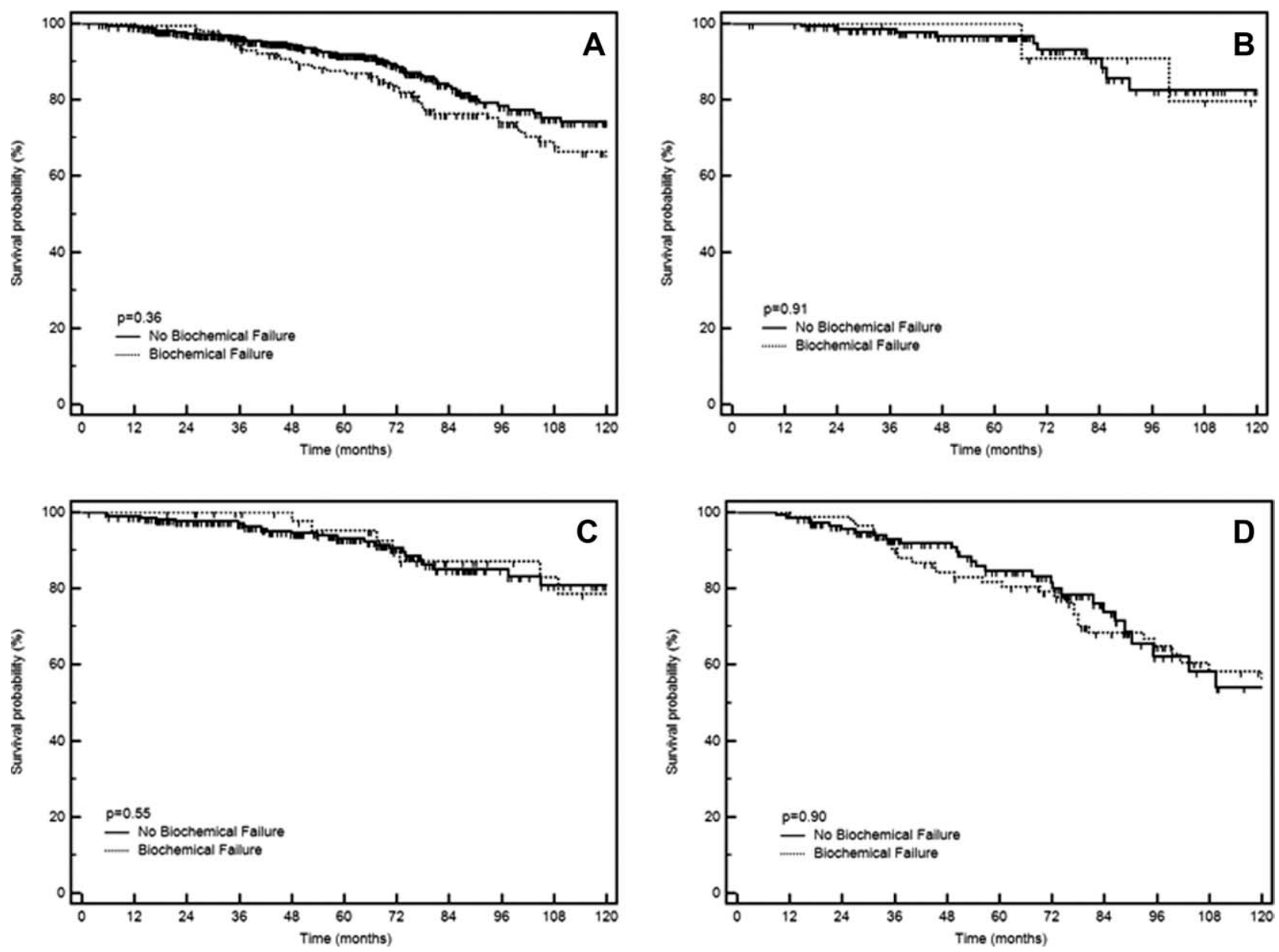

Figure 2. Biochemical failure did not predict for worse overall survival among all patients (A), and as stratified by National Comprehensive Cancer Network risk group (low risk, B; intermediate risk, C; high risk, D).

(metastasis-free rate, $79 \% \pm 8 \%$ vs $33 \% \pm 5 \% ; P<$ .0001 ; hazard ratio $[\mathrm{HR}], 4.2 ; 95 \%$ confidence interval [CI], 2.2-8.0; Fig. 3A). Similarly, $7 \% \pm 1 \%$ of patients died of PCa at 8 years, and short interval to biochemical failure predicted for worse CSS than long interval to biochemical failure or no biochemical failure. No patients without biochemical failure died of PCa; the 8-year freedom from PCa death was $87 \% \pm 4 \%$ in patients with a long interval to biochemical failure, and $44 \% \pm 9 \%$ in those with a short interval to biochemical failure $(P<$ .0001; HR, 5.6; 95\% CI, 2.4-12.9; Fig. 3B). Finally, a short interval to biochemical failure also predicted for worse OS, with an 8 -year rate of OS of $38 \% \pm 9 \%$ in those with a short interval to biochemical failure as compared with $87 \% \pm 4 \%$ in those with a long interval to biochemical failure ( $P<.0001$; HR, 4.9; 95\% CI, 2.3-10.3). Furthermore, OS among patients with a long interval to biochemical failure did not differ significantly as com- pared with those with no biochemical failure $(P>.06$; HR, 0.7; 95\% CI, 0.4-1.1; Fig. 3C).

Potential confounding factors that could limit the applicability of the interval to biochemical failure were next evaluated. The negative prognostic impact of a short interval to biochemical failure on CSS and OS was evident both in those treated with EBRT alone and those treated with EBRT plus ADT. In those treated with EBRT and no ADT, a short interval to biochemical failure carried an 8.8-fold increased risk for death from $\mathrm{PCa}$ as compared with a long interval to biochemical failure $(P<.0001$; 95\% CI, 2.2-36; Fig. 4A) and a 7.3-fold increased risk of all cause mortality $(P<.0001 ; 95 \% \mathrm{CI}, 2.0-27$; Fig. 4C). For patients who received both EBRT and ADT, a short interval to biochemical failure was associated with a 3.7fold increased risk of $\mathrm{PCa}$ death $(P=.003$; $95 \% \mathrm{CI}, 1.3$ 10.7; Fig. 4B) and a 3.3-fold increased risk of all cause mortality $(P=.0016$; $95 \%$ CI, 1.3-8.1; Fig. 4D) as 

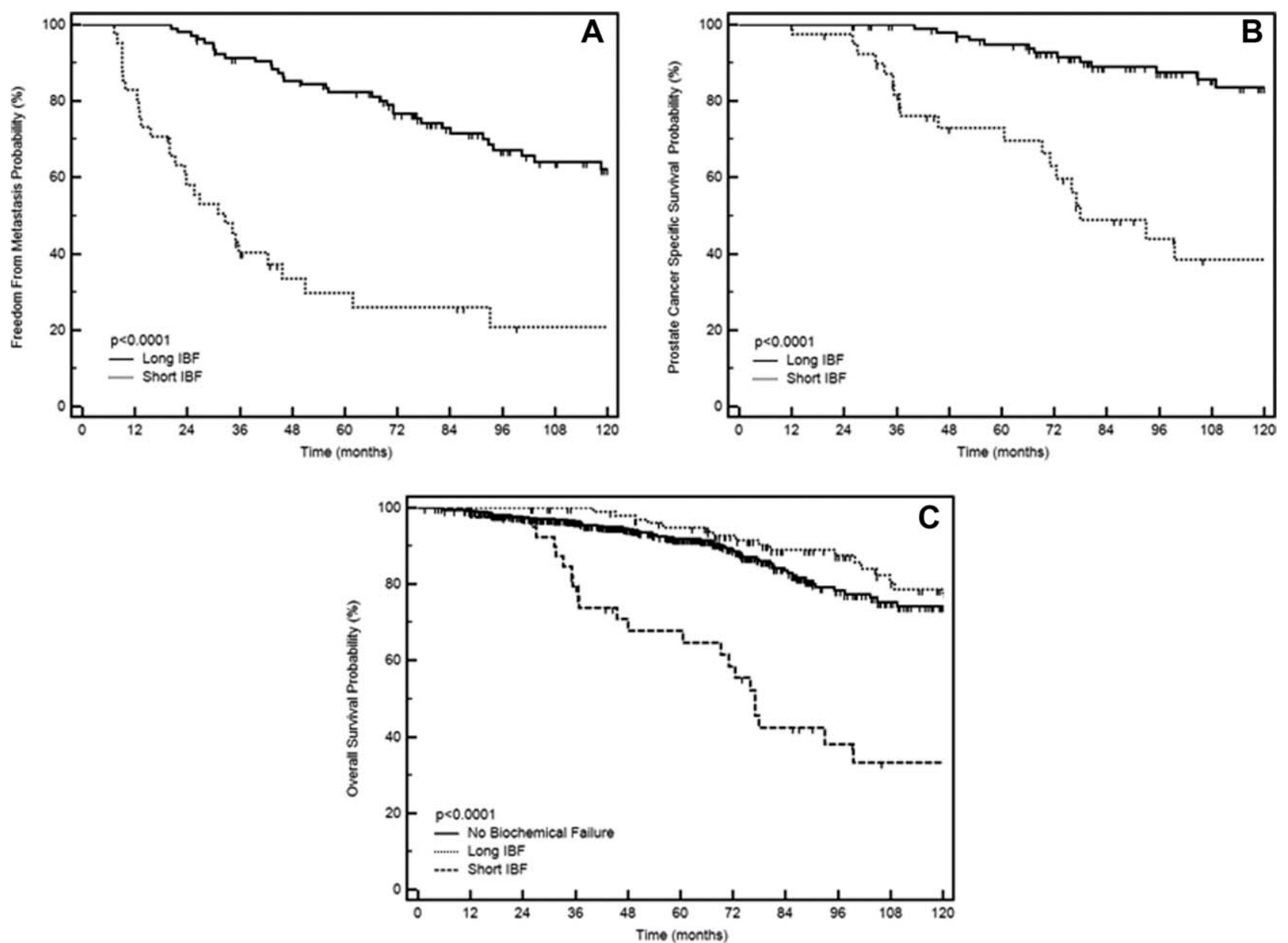

Figure 3. A short interval to biochemical failure (IBF) was associated with worse metastasis-free survival (A), cause-specific survival (B), and overall survival (C), as compared with long interval to biochemical failure or no biochemical failure.

compared with a long interval to biochemical failure. As above, patients with a long interval to biochemical failure did not have significantly different OS as compared with those without biochemical failure when treated with RT alone (Fig. 4B) or when treated with RT + ADT (Fig. 4D).

In addition, given the potential influence of age on OS, the impact of a short interval to biochemical failure was assessed as a function of age. When stratified by those $<60,60$ to 70 , or $>70$ years of age, there was a similar relation, such that in each age group a short interval to biochemical failure predicted for worse OS, whereas a long interval to biochemical failure did not (Table 2). We also considered the possibility that a short interval to biochemical failure resulted from less aggressive treatment, especially as compared with patients with a long interval to biochemical failure, and that this might explain a worse clinical outcome. However, we found no difference in RT dose based upon biochemical failure status $(P=.7$, Table 1 ) and that pelvic EBRT fields were most commonly used in patients who would later experience a short interval to biochemical failure $(P<.0001$, Table 1$)$. Patients with a short interval to biochemical failure also received adjuvant ADT more frequently than those patients with a long interval to biochemical failure $(61 \%$ vs $47 \%, P<.004)$ and were treated with a longer duration of ADT (median, 25 vs 6 months; $P<.001$ ). Finally, patients with a short interval to biochemical failure were not only more likely to receive salvage ADT after biochemical failure, but also as a group received it sooner than those with a long interval to biochemical failure $(P<.001 ; \mathrm{HR}, 2.7 ; 95 \% \mathrm{CI}$, 1.6-4.5; Fig. 5). Two years after biochemical failure, $85 \%$ $\pm 6 \%$ of those with a short interval to biochemical failure received salvage $\mathrm{ADT}$, whereas only $53 \% \pm 6 \%$ of those with a long interval to biochemical failure were accordingly treated. 

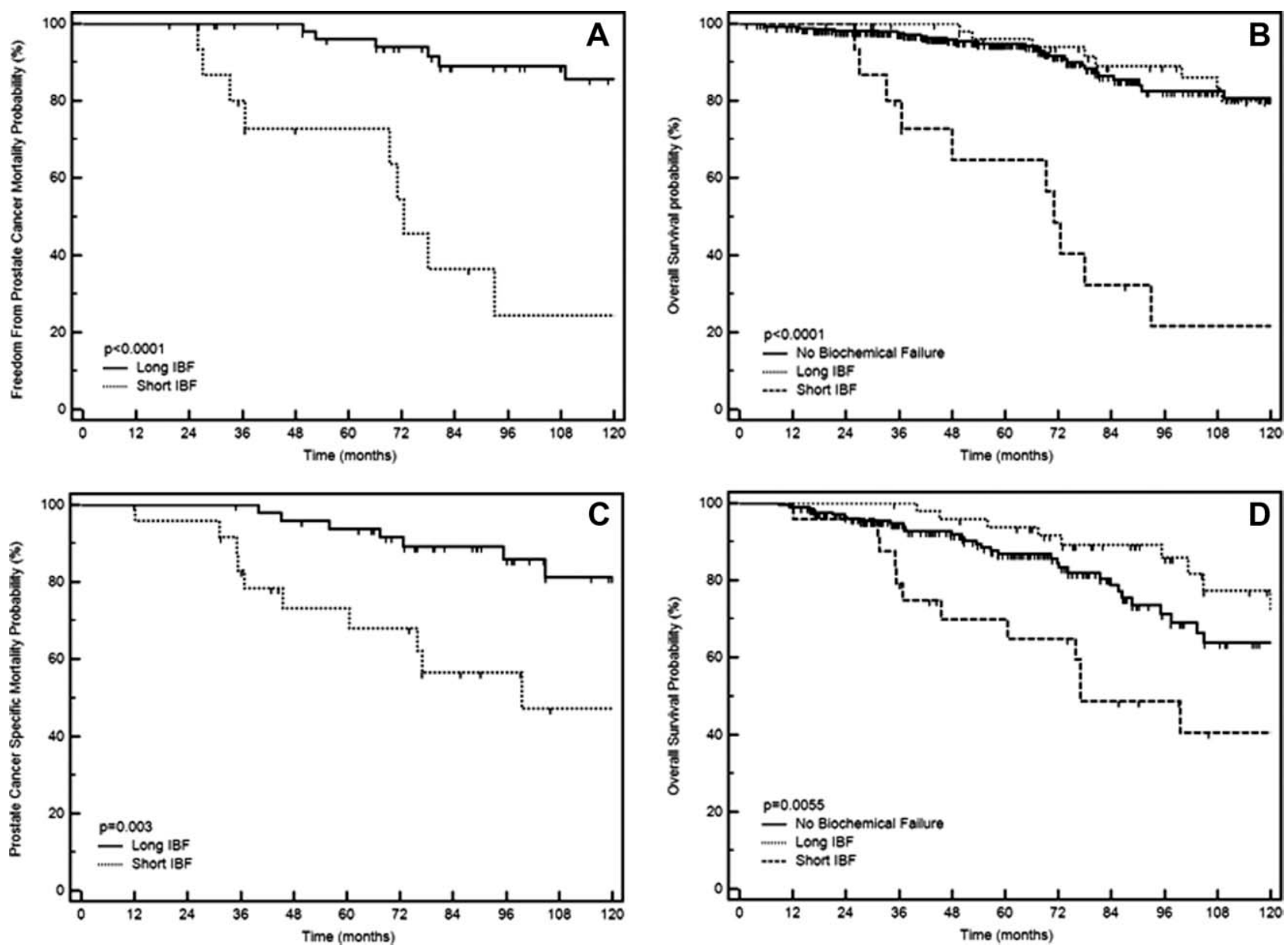

Figure 4. The negative prognostic impact of a short interval to biochemical failure (IBF) on cause-specific survival (A, C) and overall survival $(B, D)$ was evident whether patients were treated with radiation therapy (RT) alone (A, B) or with androgen deprivation therapy and RT (C, D).

Table 2. Short Interval to Biochemical Failure Predicts for Decreased Overall Survival Across All Ages

\section{Group}

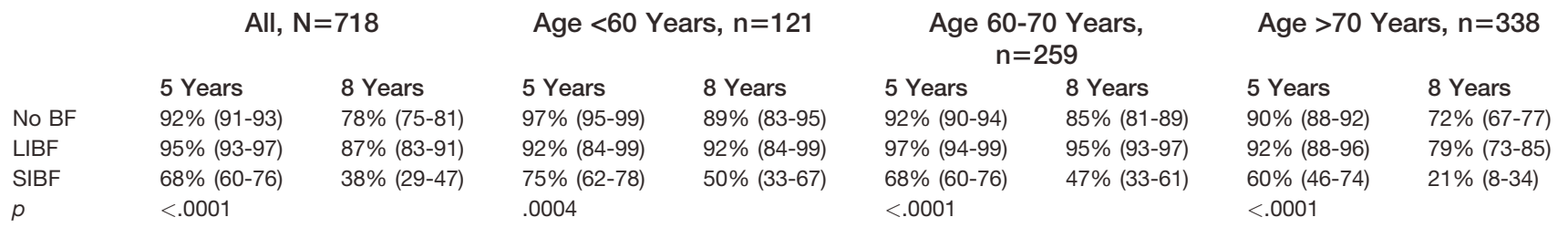

Abbreviations: BF, biochemical failure; LIBF, long interval to biochemical failure; SIBF, short interval to biochemical failure.

\section{Multivariate Regression Results}

Finally, a step-wise Cox proportional hazards regression model was used to determine which factors were prognostic for prostate-cancer specific death and overall mortality (Table 3). Patients with Gleason scores $>7$ or a short interval to biochemical failure were more likely to die of
PCa, whereas long-term ADT decreased the risk of PCarelated death. Similarly multivariate analysis of OS revealed that Gleason scores $>7$, advanced age, comorbid illness, and a short interval to biochemical failure increased the risk of all cause mortality. As above, longterm ADT decreased the risk of all cause mortality. 
Factors that were evaluated, but not prognostic for either CSS or OS, included PSA (as a continuous variable), Gleason score $\leq 7$, clinical TNM classification, the use of short-term ADT, pelvic RT, and biochemical failure $>18$ months from the end of treatment.

\section{DISCUSSION}

Given the long natural history of PCa, several biomarkers that may act as surrogates for CSS and OS have been evaluated. ${ }^{10,14,16}$ In the present study, we independently validated the interval to biochemical failure as a surrogate for metastasis-free survival and CSS in patients treated with

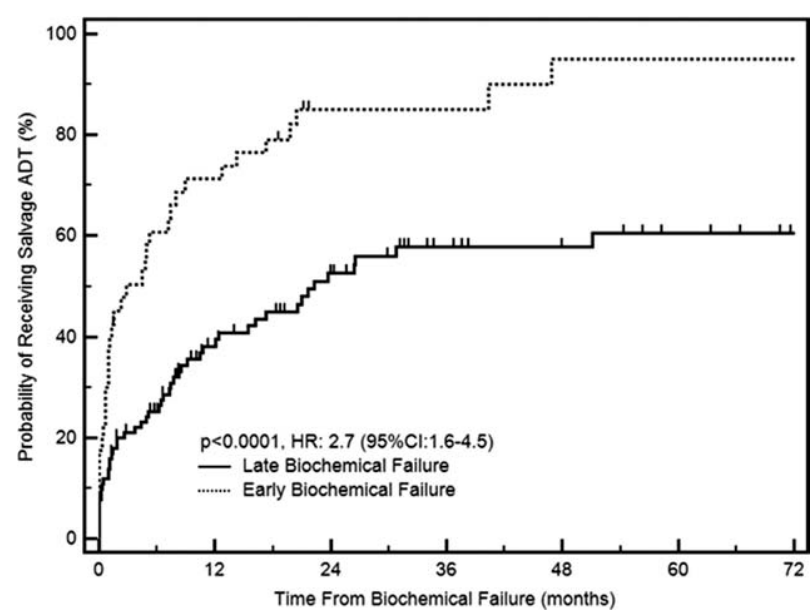

Figure 5. Patients with a short interval to biochemical failure were more likely to receive salvage androgen deprivation therapy (ADT) and received ADT earlier than those with late failures. $\mathrm{Cl}$, confidence interval; HR, hazard ratio. dose-escalated EBRT (with or without ADT), using a previously determined cutpoint of 18 months. Multivariate analysis confirmed that a short interval to biochemical failure was the strongest prognostic factor for CSS, associated with an 18-fold increase in the risk of PCa death. More importantly, multivariate analysis demonstrated that patients with a short interval to biochemical failure were $1.5 \times$ more likely to die from any cause than those without biochemical failure. This was clearly influenced by the finding that $77 \%$ of the patients treated in this cohort had intermediate-risk or high-risk disease. Interestingly, those with a long interval to biochemical failure did not have worse OS when compared with those without biochemical failure, which was confirmed upon multivariate analysis. Not surprisingly, given that most deaths in the group were not because of $\mathrm{PCa}$, patient age and the presence of comorbid illness more significantly impacted OS than did the interval to biochemical failure. From this retrospective review, were are unable to make comment as to the impact of metastatic failure versus local failure; however, given the benefit observed through the addition of RT to ADT and vice versa, it is clear that both local and systemic control are likely of importance.

The benefit of ADT in concert with EBRT has been demonstrated in multiple randomized trials, as shown by improved disease-specific (and in some studies, overall) survival in patients with locally advanced and/or highgrade PCa treated with conventional dose RT. ${ }^{18-20}$ However, the timing of ADT after biochemical failure is more controversial. The NCCN has suggested deferring salvage hormonal therapy until documented metastatic or symptomatic disease. ${ }^{2}$ Other authors, however, advocate early

Table 3. Multivariate Cox-Proportional Hazards Analysis of Cause-Specific and Overall Survival

\section{Covariate}

Gleason score 2-6

Gleason score 8

Gleason score 9-10

No ADT

LTAD therapy

No BF

Short IBF

Age $^{\mathrm{a}}$

$\mathrm{CMI}^{\mathrm{b}}$
Cause-Specific Survival

$\begin{array}{lll}p & \text { HR } & 95 \% \mathrm{Cl} \\ \begin{array}{l}\text { Reference } \\ <.04\end{array} & \\ <.0001 & 2.5 & 1.1-5.4 \\ \begin{array}{l}\text { Reference } \\ .0013\end{array} & 12.3 & 5.6-28 \\ \begin{array}{l}\text { Reference } \\ <.0001\end{array} & 0.20 & 0.07-0.52 \\ & 18.1 & 8.4-39\end{array}$

\section{Overall Survival}

$\begin{array}{ll}\text { HR } & 95 \% \mathrm{Cl} \\ & \\ 2.4 & 1.4-4.2 \\ 17.9 & 9.6-33 \\ & \\ 0.20 & 0.08-0.36 \\ & \\ 1.5 & 1.2-2.1 \\ 1.09 & 1.06-1.11 \\ 1.4 & 1.2-1.6\end{array}$

Abbreviations: ADT, androgen deprivation therapy; BF, biochemical failure; Cl, confidence interval; CMI, Charlson Comorbidity Index; HR, hazard ratio; IBF, interval to biochemical failure; LTAD, long-term androgen deprivation.

Variables evaluated but that were not prognostic included: prostate-specific antigen, TNM classification, Gleason 7, short-term ADT, the use of pelvic radiotherapy, and BF (with a long IBF).

${ }^{\text {a } A g e ~ a s ~ a ~ c o n t i n u o u s ~ v a r i a b l e ~ p e r ~ y e a r . ~}$

${ }^{\mathrm{b}} \mathrm{CMI}$ as a continuous variable per point. 
ADT use to prevent the morbidity of potentially symptomatic metastatic disease. ${ }^{21}$ Although randomized investigations have failed to show a survival benefit in upfront versus delayed use, there does appear to be a benefit to immediate (and indefinite) ADT use in at least the postoperative setting for lymph node-positive patients. ${ }^{22}$

A secondary analysis of RTOG 85-31 (which randomized patients with locally advanced PCa to EBRT alone vs EBRT with lifelong adjuvant ADT) evaluated patients allocated to the RT-alone arm and divided them into 2 groups: early salvage therapy versus late salvage therapy as defined by the PSA level at which salvage was initiated, using $10 \mathrm{ng} / \mathrm{mL}$ as a threshold. Multivariate analysis demonstrated a statistically significant improvement in OS among patients who were salvaged before their PSA level reached $10 \mathrm{ng} / \mathrm{mL}$, although interestingly no significant differences were observed in either CSS or local control. ${ }^{23}$ This suggests a possible bias toward using salvage $\mathrm{ADT}$ in those who were overall in better health. A similar secondary analysis was conducted for the RTOG 86-10 trial (which randomized patients with locally advanced PCa to EBRT alone vs EBRT + 4 months neoadjuvant and concurrent ADT). For patients randomized to RT alone (without adjuvant ADT), the initiation of salvage $\mathrm{ADT}$ before metastasis improved both $\mathrm{OS}$ and $\mathrm{CSS}^{24}$ The results of the present analysis would seem to reinforce the above findings, suggesting that patients with a short interval to biochemical failure harbor aggressive disease and may stand to benefit from early and aggressive salvage therapy.

The primary strengths of this study include the contemporary and consistent treatment of patients over a 10year period. These patients were treated in the modern era of dose-escalated EBRT, and as such the minimum PTV treatment dose was $75 \mathrm{~Gy}$. This is in contrast to the report by Buyyounouski and colleagues, where the median radiotherapy dose was $72 \mathrm{~Gy}$ and the minimum reported dose was $67 \mathrm{~Gy},{ }^{14}$ and the TROG 96.01 study, where the prescribed RT dose was 66.6 Gy. ${ }^{16}$ The dose of RT is relevant because increased dose is more likely to improve local and biochemical control and has been demonstrated to alter the timing of PSA response, which could potentially impact the relevance of a short interval to biochemical failure. ${ }^{25}$ Limitations in the current data include the retrospective analysis and the relatively few events. In addition, the duration of ADT can influence the timing of testosterone recovery, ${ }^{26}$ which in turn may delay the rise of PSA, ${ }^{27}$ thereby altering the prognostic significance of the interval to biochemical failure. Unfortunately, we were unable to control for testosterone recovery in the current analysis, as these data were not routinely obtained.

\section{Conclusions}

A short interval to biochemical failure, defined as within 18 months of completing therapy (EBRT and/or ADT), correlated with a significantly increased rate of distant metastasis, decreased CSS, and decreased OS, as compared with a long interval to biochemical failure or a lack of biochemical failure. We are encouraged that this easily determined PSA-derived endpoint predicts for OS after RT in PCa. Nevertheless, these data regarding the interval to biochemical failure are hypothesis generating and must be validated within other datasets or randomized trials before gaining widespread acceptance.

\section{FUNDING SOURCES}

No specific funding was disclosed.

\section{CONFLICT OF INTEREST DISCLOSURES}

The authors made no disclosures.

\section{REFERENCES}

1. Roach M III, Hanks G, Thames H Jr, et al. Defining biochemical failure following radiotherapy with or without hormonal therapy in men with clinically localized prostate cancer: recommendations of the RTOG-ASTRO Phoenix Consensus Conference. Int J Radiat Oncol Biol Phys. 2006; 65:965-974.

2. Scherr D, Swindle PW, Scardino PT. National Comprehensive Cancer Network guidelines for the management of prostate cancer. Urology. 2003;61:14-24.

3. Cox G, Kaplan G,for the American Society for Therapeutic Radiology and Oncology Consensus Panel. Consensus statement: Guidelines for PSA following radiation therapy. Int J Radiat Oncol Biol Phys. 1997;37:1035-1041.

4. Ray ME, Thames HD, Levy LB, et al. PSA nadir predicts biochemical and distant failures after external beam radiotherapy for prostate cancer: a multi-institutional analysis. Int J Radiat Oncol Biol Phys. 2006;64:1140-1150.

5. Horwitz EM, Vicini F, Ziaja EL, et al. Assessing the variability of outcome for patients treated with localized prostate irradiation using different definitions of biochemical control. Int J Radiat Oncol Biol Phys. 1996;36:565-571.

6. Hanlon AL, Diratzouian H, Hanks GE. Posttreatment prostate-specific antigen nadir highly predictive of distant failure and death from prostate cancer. Int J Radiat Oncol Biol Phys. 2002;2002:297-303.

7. DeWitt KD, Sandler HM, Weinberg V, et al. What does postradiotherapy PSA nadir tell us about freedom from PSA failure and progression-free survival in patients with low and intermediate-risk localized prostate cancer? Urology. 2003; 62:492-496.

8. Crook JM, Bahadur YA, Bociek RG, et al. Radiotherapy for localized prostate carcinoma: the correlation of pretreatment 
prostate specific antigen and nadir prostate specific antigen with outcome as assessed by systematic biopsy and serum prostate specific antigen. Cancer. 1997;79:328-336.

9. Lee WR, Hanlon AL, Hanks GE. Prostate specific antigen nadir following external beam radiation therapy for clinically localized prostate cancer: the relationship between nadir level and disease-free survival. J Urol. 1996;156(2 pt 1):450-453.

10. D’Amico AV, Moul J, Carroll PR, et al. Prostate specific antigen doubling time as a surrogate end point for prostate cancer specific mortality following radical prostatectomy or radiation therapy. J Urol. 2004;172:S42-S47.

11. D'Amico AV, Moul J, Carroll PR, et al. Surrogate end point for prostate cancer-specific mortality after radical prostatectomy or radiation therapy. J Natl Cancer Inst. 2003; 95:1376-1383.

12. Zelefsky MJ, Ben-Porat L, Scher HI, et al. Outcome predictors for the increasing PSA state after definitive externalbeam radiotherapy for prostate cancer. J Clin Oncol. 2006; 23:826-831.

13. Pound CR, Partin AW, Eisenberger MA, Chan DW, Pearson JD, Walsh PC. Natural history of progression after PSA elevation following radical prostatectomy. JAMA. 1999;281: 1591-1597.

14. Buyyounouski MK, Hanlon AL, Horwitz EM, Pollack A. Interval to biochemical failure highly prognostic for distant metastasis and prostate cancer-specific mortality after radiotherapy. Int J Radiat Oncol Biol Phys. 2008;70:59-66.

15. Buyyounouski MK, Pickles T, Kestin L, Allison R, Williams SG. Validating the interval to biochemical failure for the identification of potentially lethal prostate cancer. Int $J$ Radiat Oncol Biol Phys. 2009;75:S103-S104.

16. Denham JW, Stiegler A, Wilcox C, et al. Time to biochemical failure and prostate-specific antigen doubling time as surrogates for prostate cancer-specific mortality: evidence from the TROG 96.01 randomised controlled trial. Lancet Oncol. 2008;9:1058-1068.

17. Charlson ME, Pompei P, Ales KL, MacKenzie CR. A new method of classifying prognostic comorbidity in longitudinal studies: development and validation. J Chronic Dis. 1987; 40:373-383.
18. Bolla M, Collette L, Blank L, et al. Long-term results with immediate androgen suppression and external irradiation in patients with locally advanced prostate cancer (an EORTC study), a phase III randomized trial. Lancet. 2002;360:103.

19. Pilepich MV, Winter K, Lawton CA, et al. Androgen suppression adjuvant to definitive radiotherapy in prostate carcinoma-long-term results of phase III RTOG 85-31. Int J Radiat Oncol Biol Phys. 2005;61:1285-1290.

20. Roach M, Bae K, Speight J, et al. Short-term neoadjuvant androgen deprivation therapy and external-beam radiotherapy for locally advanced prostate cancer: long-term results of RTOG 8610. J Clin Oncol. 2008;26:585-591.

21. Moul JW, Banez LL, Freedland SJ. Rising PSA in nonmetastatic prostate cancer. Oncology. 2007;21:1436-1454.

22. Walsh PC, Deweese TL, Eisenberger MA. A structured debate: immediate versus deferred androgen suppression in prostate cancer-evidence for deferred treatment. J Urol. 2001;166:508-516.

23. Souhami L, Bae K, Pilepich M, Sandler H. Timing of salvage hormonal therapy in prostate cancer patients with unfavorable prognosis treated with radiotherapy: a secondary analysis of Radiation Therapy Oncology Group 85-31. Int J Radiat Oncol Biol Phys. 2010;78:1301-1306.

24. Shipley WU, Desilvio M, Pilepich MV, et al. Early initiation of salvage hormone therapy influences survival in patients who failed initial radiation for locally advanced prostate cancer: a secondary analysis of RTOG protocol 8610. Int J Radiat Oncol Biol Phys. 2006;64:1162-1167.

25. Kuban DA, Levy LB, Cheung MR, et al. Long-term failure patterns and survival in a randomized dose-escalation trial for prostate cancer. Who dies of disease? Int J Radiat Oncol Biol Phys. 2011;79:1310-1317.

26. Yoon FH, Gardner SL, Danjoux C, Morton G, Cheung P, Choo R. Testosterone recovery after prolonged androgen suppression in patients with prostate cancer. J Urol. 2008; 180:1438-1444.

27. D’Ambrosio DJ, Ruth K, Buyyounouski MK, Horwitz EM, Uzzo RG, Pollack A. Prostate specific antigen kinetics in men treated with radiotherapy and androgen deprivation. Int J Radiat Oncol Biol Phys. 2006;66:S334-S335. 